\section{A single fatal dose of olanzapine}

Sir,

A 52-year male presented with acute onset clouding of consciousness, rigidity, diaphoresis, urinary incontinence, and myoclonic jerks. He had recently developed a stress induced mood disturbance for which he was prescribed olanzapine. He had consumed a single $5 \mathrm{mg}$ dose of the drug on the previous morning. Two hours post admission he developed high rise of temperature with further deterioration of sensorium. Neurological examination revealed a Glasgow coma scale score of 6, 'lead-pipe' rigidity, generalized myoclonus, and brisk deep tendon reflexes. The serum creatinine phosphokinase (CPK) level was 8,240 U/L, (Normal 60-400 U/L) and urine was positive for myoglobin. We diagnosed the case as neuroleptic malignant syndrome, discontinued olanzapine and initiated bromocriptine and clonazepam. His serum iron level was $18 \mu \mathrm{g} / \mathrm{dl}$. Serial CPK estimation showed a gradual normalization over the next eight days but there was little improvement in his sensorium even one month after CPK normalization. Prolonged recumbence resulted in multiple pressure sores and he eventually succumbed to aspiration pneumonia eight weeks after his admission.

Neuroleptic malignant syndrome (NMS) is a potentially fatal drug induced movement disorder. Although myoclonus is only occasionally seen, ${ }^{[1]}$ our case had a prominent myoclonic element with severe rigidity. Our patient did meet the criterion for catatonia; however, it differs from the functional lethal catatonia because the latter begins usually with extreme psychotic agitation while NMS usually begins with rigidity as in our case. ${ }^{[2]}$ Hypoferremia present in $96 \%$ of cases is an important marker of this disease. ${ }^{[3]}$ Two syndromes closely resemble NMS, viz. Serotonin syndrome and lethal catatonia. But both of them do not have any temporal relation to neuroleptic therapy, which is a usual requisite for NMS.

NMS can develop when a neuroleptic is initiated, reintroduced after a drug free period or with change in dosage or potency of the drug. Two-thirds of the cases develop during the first week of drug initiation; however, there are reports of NMS in patients who have been stable on their drugs for months to years. Although among neuroleptics, haloperidol and fluphenazine are the common culprits, newer atypical antipsychotic drugs are being increasingly reported. They have come into wide clinical use due to their reduced propensity to cause extra pyramidal and tardive syndromes and almost all of them are reported to have caused NMS. Decreased serum iron is known to have a modulatory effect on dopamine receptor sensitivity. There are reports of poor benzodiazepene response in patients having low serum iron, as was the case in our patient. ${ }^{[4]}$

With the recent upsurge in the use of atypical antipsychotics, even by the primary care physicians, this potentially fatal complication should always be borne in mind as it warrants a high index of suspicion for diagnosis and early therapeutic intervention to prevent fatality and long-term morbidity.

\section{Shounak Majumder ${ }^{1}$, Sanjay K. Mandal ${ }^{2}$, Gautam Guha ${ }^{3}$, Dipanjan Bandyopadhyay², Subhasis Roy Chowdhury ${ }^{1}$}

${ }^{1}$ Department of Medicine, Medical College, Kolkata, ${ }^{2}$ Department of Medicine, Bankura Sammilani Medical College, ${ }^{3}$ Department of Neurology, Medical College Kolkata. E-mail: docshounak@yahoo.co.in

\section{References}

DOI: $10.4103 / 0028-3886.55569$

1. Weinberg S, Twerky RS. Neurolept malignant syndrome. Anesth Analg 1983;62:848-50.

2. Castillo E, Rubin RT, Holsboer-Trachsler E. Clinical differentiation between lethal catatonia and neuroleptic malignant syndrome. Am J Psychiatry 1989;146:324-8.

3. Burch EA, Montoya J. Neuroleptic malignant syndrome in an AIDS patient. J Clin Psychopharmacol 1989;9:228-9.

4. Lee J. Serum iron in catatonia and neuroleptic malignant syndrome. Biol Psychiatry 1998;44:499-507.

Accepted on 10-08-2009 